\title{
Physical Activity In Renal Disease (PAIRED) and the effect on hypertension: study protocol for a randomized controlled trial
}

Stephanie Thompson ${ }^{1 *}$, Natasha Wiebe ${ }^{1}$, Gabor Gyenes ${ }^{2}$, Rachelle Davies ${ }^{1}$, Jeyasundar Radhakrishnan ${ }^{3}$ and Michelle Graham²

\begin{abstract}
Background: The prevalence of hypertension among people with chronic kidney disease is high with over $60 \%$ of people not attaining recommended targets despite taking multiple medications. Given the health and economic implications of hypertension, additional strategies are needed. Exercise is an effective strategy for reducing blood pressure in the general population; however, it is not known whether exercise would have a comparable benefit in people with moderate to advanced chronic kidney disease and hypertension.

Methods: This is a parallel-arm trial of adults with hypertension (systolic blood pressure greater than $130 \mathrm{mmHg}$ ) and an estimated glomerular filtration rate of $15-45 \mathrm{ml} / \mathrm{min} 1.73 \mathrm{~m}^{2}$. A total of 160 participants will be randomized, with stratification for estimated glomerular filtration rate, to a 24-week, aerobic-based exercise intervention or enhanced usual care. The primary outcome is the difference in 24-h ambulatory systolic blood pressure after 8 weeks of exercise training. Secondary outcomes at 8 and 24 weeks include: other measurements of blood pressure, aortic stiffness (pulse-wave velocity), change in the Defined Daily Dose of anti-hypertensive drugs, medication adherence, markers of cardiovascular risk, physical fitness (cardiopulmonary exercise testing), 7-day accelerometry, quality of life, and adverse events. The effect of exercise on renal function will be evaluated in an exploratory analysis. The intervention is a thrice-weekly, moderate-intensity aerobic exercise supplemented with isometric resistance exercise delivered in two phases. Phase 1: supervised, facility-based, weekly and home-based sessions (8 weeks). Phase 2: home-based sessions (16 weeks).

Discussion: To our knowledge, this study is the first trial designed to provide a precise estimate of the effect of exercise on blood pressure in people with moderate to severe CKD and hypertension. The findings from this study should address a significant knowledge gap in hypertension management in CKD and inform the design of a larger study on the effect of exercise on CKD progression.
\end{abstract}

Trial registration: ClinicalTrials.gov, ID: NCT03551119. Registered on 11 June 2018.

Keywords: Exercise, Hypertension, Blood-pressure, Chronic kidney disease, Randomized trial, Ambulatory blood pressure

\footnotetext{
* Correspondence: th11@ualberta.ca

'Division of Nephrology and Immunology, 11-112R CSB, 152 University

Campus NW, University of Alberta, 11-112 Clinical Sciences Building,

Edmonton, AB T6G 2G3, Canada

Full list of author information is available at the end of the article
}

(c) The Author(s). 2019 Open Access This article is distributed under the terms of the Creative Commons Attribution 4.0 International License (http://creativecommons.org/licenses/by/4.0/), which permits unrestricted use, distribution, and reproduction in any medium, provided you give appropriate credit to the original author(s) and the source, provide a link to the Creative Commons license, and indicate if changes were made. The Creative Commons Public Domain Dedication waiver (http://creativecommons.org/publicdomain/zero/1.0/) applies to the data made available in this article, unless otherwise stated. 


\section{Background}

In people with chronic kidney disease (CKD), hypertension is an important modifiable risk factor for both cardiovascular (CV) events and progressive renal dysfunction [1-5]. Delaying the progression of CKD is important because it enables patients to live longer without significant complications from CKD (malnutrition, bone and electrolyte disorders, anemia, higher risk of $\mathrm{CV}$ events) and the need for renal replacement therapy. Despite the significance of attaining blood pressure (BP) targets, the estimated prevalence of hypertension among clinical populations with CKD remains high at $67-86 \%$ $[6,7]$. This is despite the use of multiple anti-hypertensive drugs and access to dietary counseling on low sodium intake (more than $90 \%$ of Canadian CKD clinics have a dietician on staff) [8].

Regular exercise is effective in reducing $\mathrm{BP}$ in people without CKD. BP reduction with regular aerobic exercise ranges from -3.5 to $-6.1 \mathrm{mmHg} /-2.5$ to $-3.0 \mathrm{mmHg}$ [9-11]. Although guidelines for the management of CKD recommend exercise for $\mathrm{CV}$ health, randomized trials in people with impaired estimated glomerular filtration rate (eGFR) and hypertension are limited [12, 13]. In addition, compared to those with normal kidney function, people with CKD are more likely to need multiple anti-hypertensive drugs to attain BP targets [14, 15], suggesting that responsiveness to other approaches may also be reduced. As a result of this knowledge gap, exercise resources are not offered in the routine multidisciplinary care of people with CKD and the prevalence of sedentary behavior remains double that of the general population $[8,16]$.

The primary objective of this trial is to determine the efficacy of an 8-week, aerobic-based exercise intervention on systolic blood pressure (SBP) in people with moderate to severe CKD. We hypothesize that participants allocated to the exercise intervention will achieve a significant mean reduction in SBP as measured by the "gold standard," 24-h ambulatory blood pressure (ABPM) compared to control. Results from this efficacy trial will also be used to inform the design of a larger, multi-center trial aimed at evaluating the effect of exercise on the risk of CKD progression.

\section{Objectives}

\section{Primary objective}

The primary aim of this study is to determine the efficacy of an 8-week exercise intervention on SBP in people with moderate to severe CKD.

\section{Secondary objective}

The secondary objective is to evaluate the effect of exercise on other measures of BP: aortic stiffness, changes in the required dose of anti-hypertensive drugs, markers of $\mathrm{CV}$ risk, cardiorespiratory fitness, a physical activity, quality of life (QoL), and adverse events (AEs) at 8 and 24 weeks.

\section{Tertiary objective}

The tertiary objective is to inform the design of a larger, multi-center, randomized controlled trial (RCT) examining the effect of exercise on the progression of CKD; specifically, to establish trial processes, feasibility, and to estimate the variance of the treatment effect for eGFR.

\section{Methods}

\section{Design, setting, and participants}

The flow of participants through the trial is summarized in Fig. 1. Participants will be randomized to either an aerobic-based exercise intervention or a control. Participants will be recruited from both academic and community-based clinics within Alberta Kidney Care (AKC) North. AKC North is a single, provincially funded renal program in Edmonton, AB, Canada. The University of Alberta Research Ethics Board approved this protocol (Pro00078564). The study was prospectively registered (NCT0355119). The Standard Protocol Items: Recommendations for Interventional Trials (SPIRIT) Checklist is available as an additional document (Additional file 1).

\section{Recruitment and eligibility}

A range of recruitment strategies will be employed, including direct recruitment from AKC North CKD clinics by study staff, posters, advertising through the local branch of The Kidney Foundation, and referrals from physicians and renal clinic staff. All recruitment materials and participant information has been co-developed or reviewed with a panel of patient advisors. Prior to eligibility screening, informed written consent will be obtained by the trial study coordinator from the potential participant.

\section{Inclusion}

Eligible patients are those aged 18 years or older, with hypertension, who are followed in an Edmonton-based CKD clinic with an eGFR between 15 and $44 \mathrm{ml} / \mathrm{min}$ per $1.73 \mathrm{~m}^{2}$ on two occasions in the past year. Hypertension is defined as a resting SBP greater than $130 \mathrm{mmHg}$ on screening measurement with a validated, electronic sphygmomanometer (Omron HEM-907XL (Omron Healthcare, Kyoto, Japan)), using the mean of three readings after discarding the first reading and at least one previous SBP measurement above $130 \mathrm{mmHg}$ on a separate occasion within the past 6 months (home or clinic measurement). Although there is evidence that lower SBPs are beneficial, there is still debate regarding the optimal BP target in people with CKD $[17,18]$ and a target SBP of less than $130 \mathrm{mmHg}$ is consistent with current Canadian CKD Management Guidelines [19]. Additional 


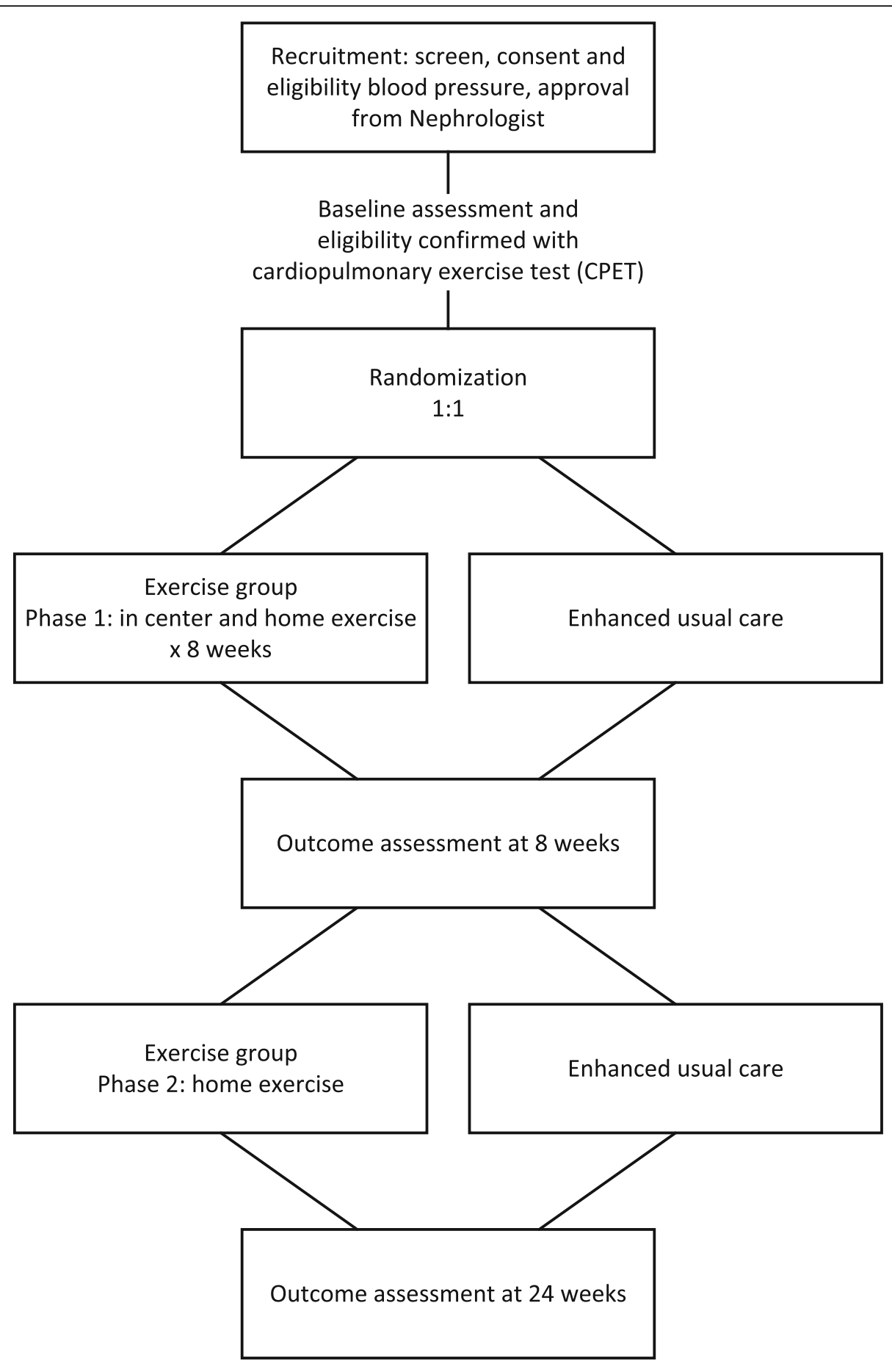

Fig. 1 Flow of participants through the trial

inclusion criteria are: able to ambulate with or without an assistive device for at least three consecutive minutes, capable of providing informed consent, English language sufficient to understand written information and comply with the testing and interventions, and approval of the attending nephrologist.

\section{Exclusion}

Resting SBP greater than $160 \mathrm{mmHg}$ or resting DBP greater than $110 \mathrm{mmHg}$ on screening $\mathrm{BP}$ clinic measurement, changes to BP medications within the past 8 weeks, arm circumference greater than $54 \mathrm{~cm}$ (size limit of large ABPM cuff), recent (within 6 weeks) or planned 
(within 6 months) major $\mathrm{CV}$ events or procedures, any absolute contraindication to exercise (American College of Sports Medicine Guidelines) [20], pregnant or planning to become pregnant within the next 9 months, life expectancy or predicted time to renal replacement therapy less than 12 months (attending physician judgment), planned move or hospital admission within the next 9 months, currently enrolled in an interventional clinical trial or a structured exercise program, significant barriers to participation (e.g., no available transportation to the training facility), solid organ transplantation, taking other medications known to affect BP (prednisone/cyclosporine) with expected adjustment in the next 9 months. Patients will be excluded from the study if exercise testing results preclude safe exercise training as defined by the American College of Cardiology/American Heart Association (ACC/ AHA) guidelines [21], e.g., abnormal BP response, early ischemic changes, and unexpected life-threatening arrhythmia. The trial cardiologists will review the electrocardiogram at peak exercise on all participants.

\section{Randomization and blinding}

Participants will be randomized (1:1) to enhanced usual care (measurement of physical activity levels) or the exercise intervention. A randomized permuted block design (of 4 and 6) will be used. To optimize comparability of the two groups at baseline, randomization will be stratified by baseline eGFR $\left(15-29 \mathrm{ml} / \mathrm{min} / 1.73 \mathrm{~m}^{2}\right.$ and $\left.30-44 \mathrm{ml} / \mathrm{min} / 1.73 \mathrm{~m}^{2}\right)$. The randomization sequence will be computer generated using Stata/MP 15.1 (https:// www.stata.com/) and allocation will be concealed by web-based central randomization using the Research Electronic Data Capture System (REDCap 8.8.2@ 2018 Vanderbilt University). Due to the nature of the intervention, participants cannot be blinded to group assignment; however, the primary outcome is objectively measured (24-h ABPM).

\section{Intervention arms \\ Exercise group}

All participants will receive standard care according to the Canadian Guidelines for the Management of Chronic Kidney Disease [19]. Those allocated to the intervention group will participate in a 24-week exercise program delivered in two phases. Phase 1 is an 8-week program of once weekly, supervised, facility-based exercise sessions and twice-weekly, home-based sessions. The weekly, facility-based sessions will take place in a rehabilitation facility at the University of Alberta Hospital. Phase 2 is a 16-week, home-based exercise program, overseen by a kinesiologist. During this phase, participants will be progressed through their home-based exercise program from phase 1 on an individualized basis. In phase 2, participants are seen once by the kinesiologist, 4 months after study start (in the middle of the 16-week home phase) and as needed. The exercise intervention is primarily aerobic, as the BP-reducing effect of exercise is greater with aerobic versus dynamic resistance exercise in people with hypertension: - $8.3(95 \% \mathrm{CI}-10.7,-6.0) / 5.2(95 \% \mathrm{CI}$ - 6.8, - 3.4) $\mathrm{mmHg}$ versus $0.47(95 \% \mathrm{CI}-4.4,5.3) /-1.0$ (95\% CI - 3.9, 1.9) $\mathrm{mmHg}$ [9]. The mode of aerobic exercise (e.g., walking, cycling) will be individualized based on participant preference and feasibility for home. In both phases, participants will be prescribed exercise on a minimum of 3 days per week at a moderate intensity (50-60\% heart rate reserve) based on the most recent cardiopulmonary exercise test (CPET). Participants will progress over phase 1 to accumulate 150 min of exercise per week [22]. Sessions may be continuous or interspersed as 10-min bouts [23-25]. In both phases, aerobic exercise will be supplemented with isometric resistance exercises (sustained muscle contraction with no change in muscle length). In both hyper- and normotensive populations, isometric resistance exercise is associated with change in SBP of -6.8 (95\% CI, -7.9 to -5.6$) \mathrm{mmHg}$ [26]. Isometric exercise is also feasible for the home setting and has shown to be safe in people with hypertension using the appropriate technique [27]. Isometric exercises will focus on large muscle groups. The period of time and the postures will be individualized and progressed over the course of the study period.

\section{Control group (enhanced usual care)}

Renal healthcare providers will continue their usual practice for all trial participants. Participants in the control group will also perform 7-day accelerometry. This is enhanced usual care because physical activity measurement is not routinely performed in CKD clinics. Accelerometry has been associated with increased levels of physical activity; however, this effect is transient and the health impact of activity monitoring is unclear [28]. Furthermore, this approach is critical for recruitment and retention in the control arm and to determine whether participants in the control arm increase their levels of physical activity. The control-arm participants will only receive their accelerometry data after they have completed the study.

\section{Adherence}

To maximize adherence to exercise in the intervention group, we will apply the core set of determinants for behavior change from Bandura's Social Cognitive Theory: knowledge of health benefits, self-efficacy (expectations about the ability to perform the behavior successfully) and outcome expectations (perceived consequences of the behavior) [29]. Strategies to increase self-efficacy for physical activity will be evidence-based (i.e., vicarious 
experience and formalized comparative feedback) [30]. The Self-efficacy for Exercise (SEE) scale will be used to evaluate participants' self-efficacy expectations of exercise expectations [31]. Throughout the trial, the kinesiologist will use motivational interviewing techniques to identify and reinforce the participant's goals and reasons for changing behavior. To assess adherence and to provide additional motivation, the trial kinesiologist will phone intervention participants every 2 weeks in phase 2. To promote retention, all participants who complete the trial will be offered an additional counseling session with the study kinesiologist and an activity-tracking device. These incentives are intended to assist control-arm participants with increasing their levels of physical activity. For members of the intervention group, these incentives are intended to support the maintenance of physical activity levels.

\section{Variables and their measurement}

\section{Baseline clinical information and study visits}

A summary of the study schedule is shown in Table 1 . Clinical data including cause of CKD, comorbidities, and medications will be collected via participant interviews, chart reviews, and reviews of clinical databases at the baseline study visit. Follow-up study visits are scheduled at 8 and 24-weeks. All participants will be contacted by phone every 2 weeks during the first 8 weeks of the trial to collect data on unscheduled visits and phone calls to healthcare providers, AEs, and changes to anti-hypertensive drugs. Over weeks 8 to 24, participants will be phoned every 4 weeks for this information. Data on AEs and medication changes will be verified using the electronic health repository.

\section{Primary outcomes}

The primary outcome for the trial is the difference in 24-h ambulatory SBP after 8 weeks of exercise training compared to control. SBP is a stronger predictor of end-stage renal disease than DBP or other measures of arterial stiffness in people with CKD [1-3, 32]. Twenty-four-hour ABPM is the gold-standard method for BP measurement and more accurately predicts renal and $\mathrm{CV}$ risk in people with CKD than clinic BP [33]. Participants will be advised to perform their regular daily routine (but not to exercise) during the 24-h ABPM period or $24 \mathrm{~h}$ before the study visit (for clinic BP measurements). ABPM will be measured using a validated device (OnTrak Ambulatory Blood Pressure Monitor 90,227-1; Spacelabs Healthcare, Mississauga, ON, Canada) [34] worn on the non-dominant arm for a $24-\mathrm{h}$ period. Readings will be obtained at 20 -min intervals from 6 a.m. to 10 p.m. and at 30-min intervals from 10 p.m. to 6 a.m. according to a recommended protocol [35].

Table 1 Overview of trial outcomes by study visit

\begin{tabular}{|c|c|c|c|c|}
\hline Outcome measure & Method & Baseline & $\begin{array}{l}\text { Study visit } 1 \\
8 \text { weeks }\end{array}$ & $\begin{array}{l}\text { Study visit } 3 \\
24 \text { weeks }\end{array}$ \\
\hline Blood pressure & $\begin{array}{l}\text { 24-h ABPM } \\
\text { Clinic BP with an automated oscillometric device }\end{array}$ & $x$ & $x$ & $x$ \\
\hline Anti-hypertensive dosing & Tabulated using the Defined Daily Dose (DDD) & $x$ & $x$ & $x$ \\
\hline Aortic stiffness & Pulse-wave velocity & $x$ & $x$ & $x$ \\
\hline Medication adherence & Questionnaire & $x$ & $x$ & $x$ \\
\hline $\begin{array}{l}\text { Markers of cardiovascular risk } \\
\text { Lipids, } \mathrm{HgA}_{1} \mathrm{C}, \mathrm{C} \text {-reactive protein } \\
\text { Albuminuria, sodium intake } \\
\text { Body composition }\end{array}$ & $\begin{array}{l}\text { Blood samples } \\
\text { Spot urine sample } \\
\text { Bioimpedance spectroscopy, BMI }\end{array}$ & $x$ & $x$ & $x$ \\
\hline Cardiorespiratory fitness & $\begin{array}{l}\text { Cardiopulmonary exercise testing } \\
\text { Six-min Walk Test }\end{array}$ & $x$ & $x$ & $x$ \\
\hline Physical activity & $\begin{array}{l}\text { 7-day accelerometry } \\
\text { IPAQ-SF }\end{array}$ & $x$ & $x$ & $x$ \\
\hline Quality of life & $\begin{array}{l}\text { KDQoL-36 } \\
\text { EQ-5D }\end{array}$ & $x$ & $x$ & $x$ \\
\hline Self-efficacy & Self-efficacy for Exercise scale & $x$ & $x$ & $x$ \\
\hline Adherence & $\begin{array}{l}\text { 7-day accelerometry } \\
\text { Participant logbook }\end{array}$ & & $x$ & $x$ \\
\hline Adverse events & Self report and health record verification & $x$ & $x$ & $x$ \\
\hline
\end{tabular}




\section{Secondary outcomes}

\begin{abstract}
Additional blood pressure and vascular outcomes The difference in overall, daytime, nighttime ABPM, and clinic BPs at 8 and 24 weeks will be reported. Clinic BPs will be measured using the mean of three readings (after discarding the first reading), each $1 \mathrm{~min}$ apart. Arterial stiffness will be estimated using the pulse-wave velocity (PWV) between the (1) carotid and femoral and the (2) carotid and radial arteries with the Complior device. Recommendations for the conditions and measurement of PWV will be followed [36]. Mean PWV will be calculated as the average of at least 10 consecutive beats in order to cover a full respiratory cycle.
\end{abstract}

Changes in anti-hypertensive drugs The Defined Daily Dose (DDD) of anti-hypertensive drugs will be used to calculate any changes to BP medications during the study [37]. Medication lists will be collected from the participant and verified with the pharmacy record (available through an electronic provincial data repository) at each study visit. At study visits, a brief questionnaire designed to capture both intentional and non-intentional medication adherence will be administered [38].

Markers of cardiovascular risk Body Mass Index (BMI), C-reactive protein, hemoglobin $\mathrm{A}_{1} \mathrm{C}\left(\mathrm{HgA}_{1} \mathrm{C}\right)$ (only participants with diabetes) and lipid profile. Urine studies include spot urinary sodium, protein, and creatinine. All samples will be collected according to routine laboratory protocol on a non-exercise day after an overnight fast. To measure fat-free mass, whole-body bioimpedance measurements will be performed using the Body Composition Monitor (BCM; Fresenius Medical Care, Germany).

Cardiorespiratory fitness Peak $\mathrm{VO}_{2}$ (volume of oxygen consumption) using a calibrated TrueOne 2400 metabolic cart (ParvoMedics TrueOne, Murray, UT, USA) from the CPET will be used to evaluate exercise-training adaptations and to determine the dose of exercise that was performed. The preferred modality for the CPET will be a stationary bicycle. After $5 \mathrm{~min}$ of rest, participants will start cycling at $10 \mathrm{~W}$ with an increasing stepped workload of $10-20 \mathrm{~W}$ every $2 \mathrm{~min}$. The CPET will be symptom-limited, with the aim of achieving a respiratory exchange ratio $>1.10$ and a Borg Rating of Perceived Exertion greater than 8 on a $0-10$ scale. $\mathrm{VO}_{2}$ will be defined as the highest oxygen uptake value recorded during the test. $\mathrm{VO}_{2}$ peak will be derived from breathby-breath indirect calorimetry and recorded as the peak 20-s average $\mathrm{VO}_{2}$ during the final minute of exercise. Using the post-CPET heart rate recovery at 1 and $5 \mathrm{~min}$, we will also explore the parasympathetic response to exercise. Other measures of functional capacity in the exercise group include the 6-min Walk Test [39], performed three times during phase 1 and once at the end of phase 2 .

Physical activity and adherence Accelerometry (Actigraph $^{\circledast}$ GT3X+ Actigraph, LLC, Pensacola, FL, USA) will be used to measure the overall difference in physical activity levels between groups and adherence in the intervention group. Participants will be instructed to wear the Actigraph device around the waist for at least 10 waking hours for seven consecutive days. Best practice and research recommendations on the management and analysis of physical activity data will be followed [40]. In phase 1 , adherence will be measured as the proportion of people who attended all eight in-center sessions within 12 weeks. For the home components of phase 1 and phase 2, adherence will be assessed as the proportion of exercise sessions completed at the prescribed frequency, time, and intensity over 7 days/number of exercise session prescribed (as measured by accelerometry). Data from participant logbooks will be used to explain any discrepancies in the accelerometry data and to provide a similar calculation of adherence overall. To determine adherence, a threshold of $70 \%$ of sessions attended and $70 \%$ of sessions performed as prescribed will be applied.

Following 7-day accelerometry, participants will complete the International Physical Activity Questionnaire Short Form (IPAQ-SF) [41]. The IPAQ-SF contains seven items that assess physical activity over the previous 7 days. Four intensity levels of activity, including sedentary behavior, are evaluated. It is the most widely used scale to monitor physical activity, thereby facilitating comparisons between populations [42].

Quality of life The Kidney Disease and Quality of Life Instrument (KDQOL-36) tool includes the 12-item Short Form Health Survey (SF-12) as generic core plus symptoms/problems of kidney disease scales [43]. The KDQOL-36 is a valid and reliable disease-specific questionnaire and has been extensively used in people with non-dialysis CKD [44]. Preference-based utilities will be measured by the EQ-5D (EuroQol Health Questionnaire) [45].

Adverse events Adverse events (AEs) are defined as any unfavorable or unintended event affecting study participants. Major AEs include death, hospitalization (unplanned), emergency department visits, CV events (stroke, myocardial infarction); doubling of serum creatinine from baseline; permanent disability; syncope, arrhythmia. Minor AEs include symptomatic hypoglycemia, hyper- or hypotension requiring medical attention, musculoskeletal 
injury, pre-syncope during or within $1 \mathrm{~h}$ after exercise. Due to the low risk of the intervention we will not use a data safety and monitoring board. All serious adverse events will be forwarded to a physician who is not involved in the trial. The physician will adjudicate whether the event was due to the exercise treatment, facilitate the necessary medical follow-up, and determine whether participation should be modified.

Feasibility criteria To inform the design of a future trial on the effect of exercise on the progression of CKD, we will evaluate enrollment, adherence, drop out and missing data using the following criteria: $30 \%$ of people screened are randomized [46] drop out less than 20\%, missing outcome data less than $10 \%$, and adherence to exercise sessions greater than 70\% (as described above).

Exploratory eGFR using the Chronic Kidney Disease Epidemiology Collaboration (CKD-EPI) equation for creatinine and cystatin $C[47,48]$.

\section{Sample size}

The sample size calculations for this trial are based on data from exercise and lifestyle modification trials in hypertensive populations [49-51]. As a 5-mmHg reduction in BP in high-risk patients reduces $\mathrm{CV}$ events by $15 \%$ [52], this study is powered to detect a clinically important difference in mean SBP of $5 \mathrm{mmHg}$ between the intervention and the control groups. Using a two-sample $t$ test, alpha 0.05 , beta 0.2 , and assuming a common standard deviation of $10 \mathrm{mmHg}, 128$ patients are required. Assuming 20\% drop out, 160 patients will be enrolled (80 per group). This sample size is also powered to detect a clinically meaningful difference of 5 points in the KDQOL-36 (alpha 0.05, beta 0.2, and assuming a common standard deviation of 10 points) [53].

\section{Statistical analysis}

All analyses will follow the intent-to-treat principle. The difference in mean 24-h ambulatory SBP between groups at 8 weeks (primary outcome) will be analyzed using a mixed linear-regression model including fixed-effects terms for time point ( 8 weeks, 24 weeks), intervention, their interaction, baseline eGFR, baseline 24-h systolic $\mathrm{ABPM}$, and a random-effects term for participant. In the primary analysis, missing values will be multiply imputed (100 iterations), using a hot-deck approach drawing all 8 -week values from the control group with replacement, within strata of age and gender [54, 55]. Estimates from the 100 datasets will be combined using Rubin's method [56]. Complete-case results, per-protocol and last-valuecarried-forward results will be presented as sensitivity analyses. Estimates and corresponding 95\% confidence intervals (CIs) will be reported for the overall difference at week 8 (and 24 weeks), adjusting for baseline eGFR and BP. All continuous outcomes with repeated measurements will be examined using mixed linear regression, following an outline similar to above. Continuous measurements without repeated measurements will be examined using linear regression, adjusting for baseline value. Residual, leverage and influence diagnostics will be examined. $P$ values $<0.05$ will be considered statistically significant. Adverse events will be tabulated, and compared between groups using Fisher's exact test. All analyses will be completed in Stata/MP 15.1 (www.stata.com/).

\section{Subgroup analyses}

Using the model from the primary analysis, the relation between exercise and eGFR will be explored. Age and gender and corresponding treatment interactions will also be examined. Proteinuria will be considered in exploratory analyses. Changes in cardiorespiratory fitness and BMI as potential mediators of BP change will be explored.

\section{Data monitoring and quality assurance}

Data will be entered directly at the site of data collection using tablets and an online RedCap database application. RedCap uses a secure syncing process and robust data validation techniques. The study coordinator will generate a weekly patient flow diagram reporting accrual, withdrawals, and fully completed protocols. The study coordinator will generate quality assurance logs containing information about participants' missing or aberrant outcome testing and will then follow-up with the pertinent study personnel or participant.

\section{Discussion}

Additional strategies to address the burden of hypertension in people with CKD are needed. Exercise is an appealing intervention due its proven efficacy in the general population and because of the potential for additional benefits. For renal healthcare providers, prescribing exercise to treat hypertension is a paradigm shift away from the mainstay of treatment, medication adjustment with or without dietary counseling on sodium intake. Therefore, to inform clinical decision-making on hypertension management in CKD, high-quality RCTs are needed to change practice. To our knowledge, this study is the first RCT designed to provide a precise estimate of the BP-lowering effect of exercise in people with moderate to severe CKD and hypertension. Strengths of this study include ABPM, which provides more accurate assessment of overall BP and kidney prognosis than clinic BPs $[57,58]$ and measurement of important confounders, such as anti-hypertensive dosing, medication adherence, and differences in physical activity levels between groups. Additional data on inflammation, vascular stiffness, and exercise recovery will improve our 
understanding of the $\mathrm{CV}$ response to exercise in people with hypertension and CKD.

This trial will also provide information on the effect of exercise on QoL as measured by the KDQoL. Developing care models that are more consistent with patient values and preferences and that address kidney-disease-specific symptoms (e.g., fatigue, low energy, depression) is a priority for decision-makers and patients [59, 60]. Exercise has improved several physical domains of QoL in people with CKD as measured with a generic instrument, but no trial has used a kidney-disease-specific tool [61, 62].

Towards the aim of designing a larger, multi-center trial to evaluate the effect of exercise on the progression of CKD, we will use the results from this current proposal to clarify trial processes, establish feasibility, and to estimate the variance of the treatment effect for eGFR. The need to identify lifestyle changes (e.g., exercise, reduced stress) that may delay kidney disease progression is an important question for patients [60]. The link between hypertension and renal function is well established and forms the scientific basis for first evaluating whether exercise can modify BP. Slower rates of eGFR decline were reported in a CKD cohort study comparing physically active people those who are sedentary $(2.8 \%$ difference in annual eGFR decline) [63]. However, RCTs with adequate sample sizes and follow-up are needed.

\section{Additional file}

Additional file 1: Recommendations for Interventional Trials (SPIRIT) 2013 Checklist. (DOC 120 kb)

\section{Abbreviations}

ABPM: Ambulatory blood pressure monitoring; AE: Adverse event; BP: Blood pressure; CKD: Chronic kidney disease; CKD-EPI: Chronic Kidney Disease Epidemiology Collaboration; CPET: Cardiopulmonary exercise test; CV: Cardiovascular; DBP: Diastolic blood pressure; eGFR: Estimated glomerular filtration rate; EQ-5D: EuroQol Health Questionnaire; $\mathrm{HgA}_{1} \mathrm{C}$ : Hemoglobin $\mathrm{A}_{1} \mathrm{C}$; KDQOL-36: The Kidney Disease and Quality of Life Instrument; PWV: Pulsewave velocity; QoL: Quality of life; RCT: Randomized controlled trial; SBP: Systolic blood pressure; SEE scale: Self-efficacy for Exercise scale; $\mathrm{VO}_{2}$ : Volume of oxygen consumption

\section{Acknowledgements}

The authors of this report are grateful to our study coordinator, Sue Szigety and our patient advisors, Steve Cutting, Marty McLeod, and Sonia Dhaliwal.

\section{Funding}

This study is funded by The Canadian Institute of Health Research (CIHR). The funders had no role in the design, collection, analysis, interpretation of the data, or writing of this protocol.

\section{Availability of data and materials}

The data collected during the trial will not be made publicly available. An anonymous minimal dataset will be made available through requests sent to the lead author.

\section{Trial status}

The current version is Version 8: 3 January 2019. Recruitment will start on 3 December 2018 and will aim to complete on 30 March 2020.

\section{Authors' contributions}

Authorship followed ICMJE guidelines. ST was responsible for the inception and design of the project and prepared the manuscript. NW, GG, RD, JR, and MG contributed to the design of the study, provided methodological input, and contributed to manuscript development. All authors read and approved the final manuscript.

Ethics approval and consent to participate

The Health Research Ethics Board at the University of Alberta approved this study (Pro00078564).

\section{Consent for publication}

Not applicable.

\section{Competing interests}

The authors declare that they have no competing interests.

\section{Publisher's Note}

Springer Nature remains neutral with regard to jurisdictional claims in published maps and institutional affiliations.

\section{Author details}

${ }^{1}$ Division of Nephrology and Immunology, 11-112R CSB, 152 University Campus NW, University of Alberta, 11-112 Clinical Sciences Building, Edmonton, AB T6G 2G3, Canada. ${ }^{2}$ Department of Cardiology, Mackenzie Health Science Centre, 8440-112 Street, Edmonton, AB T6G 2B7, Canada. ${ }^{3}$ Innovation Physical Therapy, 1582397 Street NW \#209, Edmonton, AB T5X 0C7, Canada.

Received: 20 November 2018 Accepted: 29 January 2019

Published online: 08 February 2019

\section{References}

1. Klag M, Whelton P, Randall B. Blood pressure and end-stage renal disease in men. New Engl J Med. 1996;334:13-8.

2. Young $\mathrm{JH}$. Blood pressure and decline in kidney function: findings from the Systolic Hypertension in the Elderly Program (SHEP). J Am Soc Nephrol. 2002;13:2776-82.

3. Schaeffner ES, Kurth T, Bowman TS, Gelber RP, Gaziano JM. Blood pressure measures and risk of chronic kidney disease in men. Nephrol Dial Transplant. 2008;23:1246-51.

4. Lozano R, Naghavi M, Foreman K, Lim S, Shibuya K, Aboyans V, et al. Global and regional mortality from 235 causes of death for 20 age groups in 1990 and 2010: a systematic analysis for the Global Burden of Disease Study 2010. Lancet. 2012:380:2095-128.

5. Anderson AH, Yang W, Townsend RR, Pan Q, Chertow GM, Kusek JW, et al. Time-updated systolic blood pressure and the progression of chronic kidney disease: a cohort study. Ann Intern Med. 2015;162:258-65 NIH Public Access.

6. Sarafidis PA, Li S, Chen S-C, Collins AJ, Brown WW, Klag MJ, et al. Hypertension awareness, treatment, and control in chronic kidney disease. Am J Med. 2008:121:332-40.

7. Muntner P, Anderson A, Charleston J, Chen Z, Ford V, Makos G, et al. Hypertension awareness, treatment, and control in adults with CKD: results from the Chronic Renal Insufficiency Cohort (CRIC) Study. Am J Kidney Dis. 2010;55:441-51 NIH Public Access.

8. Levin A, Steven S, Selina A, Flora A, Sarah G, Braden M. Canadian chronic kidney disease clinics: a national survey of structure, function and models of care. Can J Kidney Heal Dis. 2014;1:29.

9. Cornelissen VA, Smart NA. Exercise training for blood pressure: a systematic review and meta-analysis. J Am Heart Assoc. 2013;2:e004473.

10. Dickinson HO, Mason JM, Nicolson DJ, Campbell F, Beyer FR, Cook JV, et al. Lifestyle interventions to reduce raised blood pressure : a systematic review of randomized controlled trials. J Hypertens. 2006;24:215-33.

11. Whelton SP, Chin A, Xin X, He J. Effect of aerobic exercise on blood pressure: a meta-analysis of randomized, controlled trials. Ann Intern Med. 2002:136:493-503.

12. Howden EJ, Coombes JS, Strand H, Douglas B, Campbell KL, Isbel NM. Exercise training in CKD: efficacy, adherence, and safety. Am J Kidney Dis. 2015;65:583-91 Elsevier Inc. 
13. Heiwe $\mathrm{S}$, Jacobson $\mathrm{SH}$. Exercise training in adults with CKD: a systematic review and meta-analysis. Am J Kidney Dis. 2014;64:383-93 Elsevier Inc.

14. Plantinga LC, Miller ER, Stevens LA, Saran R, Messer K, Flowers N, et al. Blood pressure control among persons without and with chronic kidney disease: US trends and risk factors 1999-2006. Hypertension. 2009;54:47-56.

15. Unni S, White K, Goodman M, Ye X, Mavros P, Bash LD, et al. Hypertension control and antihypertensive therapy in patients with chronic kidney disease. Am J Hypertens. 2015;28:814-22 Oxford University Press.

16. Beddhu S, Baird BC, Zitterkoph J, Neilson J, Greene T. Physical activity and mortality in chronic kidney disease (NHANES III). Clin J Am Soc Nephrol. 2009:4:1901-6 American Society of Nephrology.

17. Covic A, Apetrii M, Goldsmith D, Kanbay M. SPRINT: the study nephrologists might take with a grain of salt. J Clin Hypertens. 2016;18:1185-8.

18. SPRINT Research Group, Wright JT, Williamson JD, Whelton PK, Snyder JK, Sink KM, et al. A randomized trial of intensive versus standard bloodpressure control. N Engl J Med. 2015;373:2103-16.

19. Levin A, Hemmelgarn B, Culleton B, Tobe S, McFarlane P, Ruzicka M, et al. Guidelines for the management of chronic kidney disease. Can Med Assoc J. 2008;179:1154-62.

20. Pescatello LS, Arena R, Riebe D, Thompson PD. ACSM's guidelines for exercise testing and prescription, 9th ed. Philadelphia: Lippincott Williams \& Wilkins; 2014

21. Fletcher GF, Ades PA, Kligfield P, Arena R, Balady GJ, Bittner VA, et al. Exercise standards for testing and training. Circulation. 2013;128:873-934.

22. Tremblay MS, Warburton DER, Janssen I, Paterson DH, Latimer AE, Rhodes RE, et al. New Canadian physical activity guidelines. Appl Physiol Nutr Metab. 2011;36:36-46.

23. Guidry MA, Blanchard BE, Thompson PD, Maresh CM, Seip RL, Taylor AL, et al. The influence of short and long duration on the blood pressure response to an acute bout of dynamic exercise. Am Heart J. 2006;151:1322.e5-1322. e12.

24. Jones H, Taylor CE, Lewis NCS, George K, Atkinson G. Post-exercise blood pressure reduction is greater following intermittent than continuous exercise and is influenced less by diurnal variation. Chronobiol Int. 2009;26: 293-306.

25. Bhammar DM, Angadi SS, Gaesser GA. Effects of fractionized and continuous exercise on 24-h ambulatory blood pressure. Med Sci Sport Exerc. 2012;44:2270-6.

26. Inder JD, Carlson DJ, Dieberg G, Hess NC, Smart NA. Isometric exercise training for blood pressure management: a systematic review and meta-analysis to optimize benefit. Hypertens Res. 2016;39:88-94 Nature Publishing Group.

27. Millar PJ, Bray SR, McGowan CL, MacDonald MJ, McCartney N. Effects of isometric handgrip training among people medicated for hypertension: a multilevel analysis. Blood Press Monit. 2007;12:307-14.

28. Lewis ZH, Lyons EJ, Jarvis JM, Baillargeon J. Using an electronic activity monitor system as an intervention modality: a systematic review. BMC Public Health. 2015;15:585 BioMed Central.

29. Bandura A. Health promotion by social cognitive means. Heal Educ Behav. 2004;31:143-64.

30. Ashford S, Edmunds J, French DP. What is the best way to change selfefficacy to promote lifestyle and recreational physical activity? A systematic review with meta-analysis. Br J Health Psychol. 2010;15:265-88.

31. Resnick B, Palmer MH, Jenkins LS, Spellbring AM. Path analysis of efficacy expectations and exercise behaviour in older adults. J Adv Nurs. 2000;31: 1309-15 Blackwell Science Ltd.

32. Agarwal R. Blood pressure components and the risk for end-stage renal disease and death in chronic kidney disease. Clin J Am Soc Nephrol. 2009:4:830-7.

33. Minutolo R, Agarwal R, Borrelli S, Chiodini P, Bellizzi V, Nappi F, et al. Prognostic role of ambulatory blood pressure measurement in patients with nondialysis chronic kidney disease. Arch Intern Med. 2011;171:2873-8 American Medical Association.

34. O'Brien E, Mee F, Atkins N, O'Malley K. Accuracy of the SpaceLabs 90207 determined by the British Hypertension Society protocol. J Hypertens. 1991; 9:573-4.

35. Leung AA, Daskalopoulou SS, Dasgupta K, McBrien K, Butalia S, Zarnke KB, et al. Hypertension Canada's 2017 guidelines for diagnosis, risk assessment, prevention, and treatment of hypertension in adults. Can J Cardiol. 2017;33: 557-76

36. Laurent S, Cockcroft J, Van Bortel L, Boutouyrie P, Giannattasio C, Hayoz D, et al. Expert consensus document on arterial stiffness: methodological issues and clinical applications. Eur Heart J. 2006;27:2588-605.
37. World Health Organization Collaborating Centre for Drug Statistics Methodology_Definition and general considerations. [cited 8 Aug 2017]. Available from: https://www.whocc.no/atc_ddd_index/. Accessed 8 Aug 2017.

38. Hsu KL, Fink JC, Ginsberg JS, Yoffe M, Zhan M, Fink W, et al. Self-reported medication adherence and adverse patient safety events in CKD. Am J Kidney Dis. 2015;66:621-9 NIH Public Access.

39. Crapo RO, Casaburi R, Coates AL, Enright PL, Maclntyre NR, McKay RT, et al. ATS statement: Guidelines for the six-minute walk test. Am J Respir Crit Care Med. 2002;166:111-7.

40. Migueles $\mathrm{JH}$, Cadenas-Sanchez C, Ekelund U, Delisle Nyström C, MoraGonzalez J, Löf M, et al. Accelerometer data collection and processing criteria to assess physical activity and other outcomes: a systematic review and practical considerations. Sport Med. 2017:47:1821-45.

41. International Physical Activity Questionnaire. [cited 29 June 2017]. Available from: http://www.ipaq.ki.se. Accessed 29 June 2017.

42. Lee PH, Macfarlane DJ, Lam TH, Stewart SM. Validity of the International Physical Activity Questionnaire Short Form (IPAQ-SF): a systematic review. Int J Behav Nutr Phys Act. 2011:8:115 BioMed Central.

43. Hays RD, Kallich JD, Mapes DL, Coons SJ, Carter WB. Development of the kidney disease quality of life (KDQOL) instrument. Qual Life Res. 1994;3:329-38.

44. Aiyegbusi OL, Kyte D, Cockwell P, Marshall T, Gheorghe A, Keeley T, et al. Measurement properties of patient-reported outcome measures (PROMs) used in adult patients with chronic kidney disease: a systematic review. PLoS One. 2017;12:e0179733 Eller K, editor.

45. EuroQol Group. EQ-5D: a standardised instrument for use as a measure of health outcome. [cited 24 Aug 2017]. Available from: https://euroqol.org/. Accessed 24 Aug 2017.

46. Cooper $\mathrm{CL}$, Hind D, Duncan R, Walters $\mathrm{S}$, Lartey $\mathrm{A}$, Lee $\mathrm{E}$, et al. A rapid review indicated higher recruitment rates in treatment trials than in prevention trials. J Clin Epidemiol. 2015;68:347-54.

47. Levey AS, Stevens LA, Schmid CH, Zhang YL, Castro AF, Feldman HI, et al. A new equation to estimate glomerular filtration rate. Ann Intern Med. 2009; 150:604-12.

48. Inker LA, Eckfeldt J, Levey AS, Leiendecker-Foster C, Rynders G, Manzi J, et al. Expressing the CKD-EPI (Chronic Kidney Disease Epidemiology Collaboration) cystatin C equations for estimating GFR with standardized serum cystatin C values. Am J Kidney Dis. 2011;58:682-4 NIH Public Access.

49. Vollmer WM, Appel LJ, Svetkey LP, Moore TJ, Vogt TM, Conlin PR, et al. Comparing office-based and ambulatory blood pressure monitoring in clinical trials. J Hum Hypertens. 2005;19:77-82.

50. Dimeo F, Pagonas N, Seibert F, Arndt R, Zidek W, Westhoff TH. Aerobic exercise reduces blood pressure in resistant hypertension. Hypertension. 2012:60:653-8.

51. Guimaraes GV, de Barros Cruz LG, Fernandes-Silva MM, Dorea EL, Bocchi EA. Heated water-based exercise training reduces 24-hour ambulatory blood pressure levels in resistant hypertensive patients: a randomized controlled trial (HEx trial). Int J Cardiol. 2014:172:434-41.

52. The Blood Pressure Lowering Treatment Trialists' Collaboration. Blood pressure-lowering treatment based on cardiovascular risk: a meta-analysis of individual patient data. Lancet. 2014;384:591-8 Elsevier Ltd.

53. Norman GR, Sloan JA, Wyrwich KW. Interpretation of changes in healthrelated quality of life: the remarkable universality of half a standard deviation. Med Care. 2003:41:582-92.

54. Appel LJ, Champagne CM, Harsha DW, Cooper LS, Obarzanek E, Elmer PJ, et al. Effects of comprehensive lifestyle modification on blood pressure control. JAMA. 2003;289:2083-93.

55. Andridge RR, Little RJA. A review of Hot Deck Imputation for survey nonresponse. Int Stat Rev. 2010;78:40-64.

56. Rubin DB. Multiple imputation for nonresponse in surveys. New York: Wiley; 1987

57. Agarwal R, Andersen MJ. Prognostic importance of ambulatory blood pressure recordings in patients with chronic kidney disease. Kidney Int. 2006:69:1175-80.

58. Andersen MJ, Khawandi W, Agarwal R. Home blood pressure monitoring in CKD. Am J Kidney Dis. 2005;45:994-1001.

59. Collister D, Russell R, Verdon J, Beaulieu M, Levin A. Perspectives on optimizing care of patients in multidisciplinary chronic kidney disease clinics. Can J Kidney Heal Dis. 2016;3:32 Canadian Journal of Kidney Health and Disease.

60. Hemmelgarn BR, Pannu N, Ahmed SB, Elliott MJ, Tam-Tham H, Lillie E, et al. Determining the research priorities for patients with chronic kidney disease not on dialysis. Nephrol Dial Transplant. 2017;32:847-54 Oxford University Press. 
61. Rossi AP, Burris DD, Lucas FL, Crocker GA, Wasserman JC. Effects of a renal rehabilitation exercise program in patients with CKD: a randomized, controlled trial. Clin J Am Soc Nephrol. 2014;9:2052-8 American Society of Nephrology.

62. Headley S, Germain M, Wood R, Joubert J, Milch C, Evans E, et al. Short-term aerobic exercise and vascular function in CKD stage 3: a randomized controlled trial. Am J Kidney Dis. 2014;64:222-9 Elsevier Inc.

63. Robinson-Cohen C, Littman AJ, Duncan GE, Weiss NS, Sachs MC, Ruzinski J, et al. Physical activity and change in estimated GFR among persons with CKD. J Am Soc Nephrol. 2014;25:399-406.

- fast, convenient online submission

- thorough peer review by experienced researchers in your field

- rapid publication on acceptance

- support for research data, including large and complex data types

- gold Open Access which fosters wider collaboration and increased citations

- maximum visibility for your research: over $100 \mathrm{M}$ website views per year

At $\mathrm{BMC}$, research is always in progress. 